\title{
Experiments in Autonomous Underground Guidance
}

\author{
S. Scheding, E. M. Nebot, M. Stevens and H. Durrant-Whyte \\ Department of Mechanical Engineering \\ The University of Sydney, NSW 2006, Australia. \\ e-mail: scheding/nebot/michael/hugh@tiny.me.su.OZ.AU
}

\author{
J. Roberts and P. Corke \\ CRC for Mining technology and Equipment \\ PO Box 883, Kenmore, Q 4069 \\ e-mail: jmr/pic@brb.dmt.csiro.au \\ J. Cunningham and B. Cook \\ Engineering Research Department \\ Mount Isa Mines, Mount Isa, Q 4825
}

\begin{abstract}
This paper presents the results of an experimental program for evaluating sensors and sensing technologies in an underground mining applications. The objective of the experiments is to infer what combinations of sensors will provide reliable navigation systems for autonomous vehicles operating in a harsh underground environment. Results from a wide range of sensors are presented and analysed. Conclusion as to a best combination of sensors are drawn.
\end{abstract}

\section{Introduction}

In this paper we present the work done at an operational underground mine to determine which sensors or combinations of sensors offer a solution to providing a robust navigation system for underground mining equipment. There are two main reasons for performing the trials. Firstly, automation potential to increases productivity and hence reduce mine operating costs. The second reason, is that automation (particularly in underground environments) takes people away from potentially hazardous areas. Cost reductions will arise from productivity increases, throughput time reduction and quality improvements. In environments too hazardous for human intervention, automation will be the only available solution provided it is robust and the costs involved do not affect the profits expected from the mine. Vehicle automation requires that the vehicle be controlled in order to execute a predetermined path. The vehicle may also be required to be able to dynamically avoid obstacles and provide continuous system safety monitoring. To do any of this, however, the position of the vehicle must be known at all times, requiring that the environment be sensed in some way. Existing mine automation schemes [1][2][4] require too much infrastructure to operate costeffectively, so the sensors chosen for the trial were chosen with the constraint of minimum infrastructure in mind. The sensors described in the following sections were trialed over a two week period in an underground mine on a Load, Haul and Dump truck (LHD).

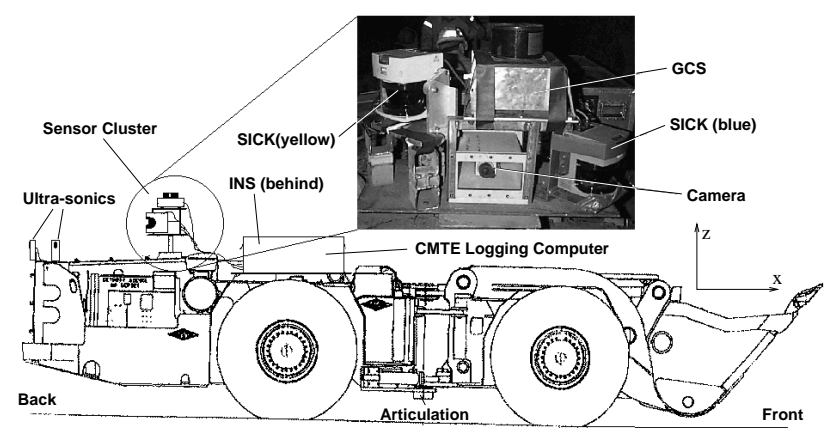

Figure 1. LHD used for the trials.

The sensors used in the trials were:

- A strap-down Inertial Navigation System (INS).

- Encoders.

- A bearing only laser scanner.

- Two time-of-flight range and bearing laser scanners.

- Two sets of ultrasonic sensors.

- A video camera and structured light sources.

It is important to realise that the task of navigating a vehicle through a mine will almost certainly require the use of multiple sensors of varying types. The sensors need redundancy if faults in the system are to be detected, and the information provided by the sensors needs to be fused in a sensible and reliable way. Algorithms such as the Kalman filter are useful for this purpose.

In this paper, section 2 provides an overview of the experimental setup used in the field trials. Section 3 discusses the results obtained from the various dead reckoning sensors, while section 4 examines the external sensors used. Finally, section 5 presents the conclusions and recommendations drawn from this set of experiments.

\section{Experimental Setup}

The data from the different sensors needed to be recorded so that it could be post-processed. To this end, a distributed network of computers was located on an LHD, synchronised by a time stamp sent from the master computer to the slaves at a rate of $50 \mathrm{~Hz}$. This enabled the data from each sensor to be individually time stamped. This is important as some of the sensors produce data 
asynchronously. The overall system os described in Figure 2.

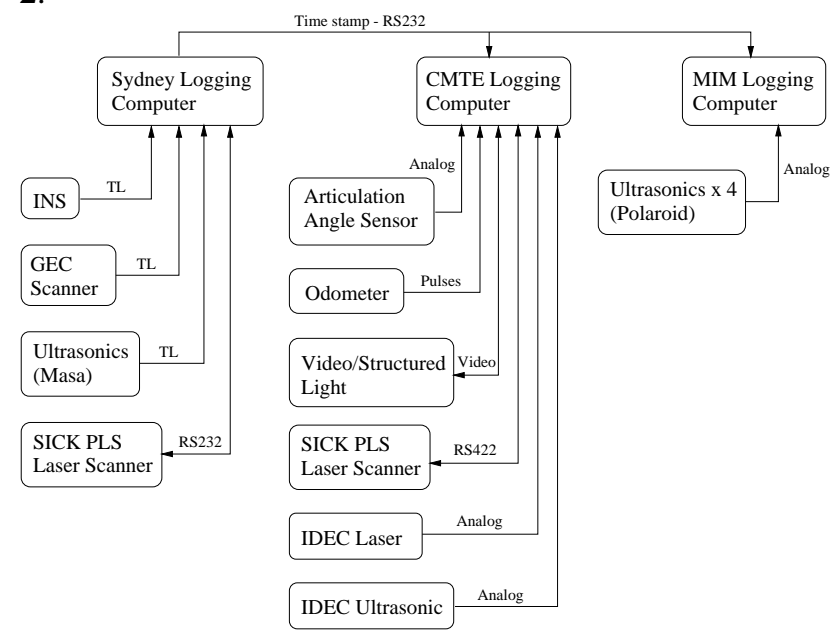

Figure 2. Data logging system.

The sensors used in the trial programme may be broken down into two broad categories, dead reckoning sensors, and external sensors. While dead reckoning sensors tend to be very robust, they accumulate error with time, so that in practise they must be periodically reset using information from the external sensors. The external sensors provide absolute information referenced to the environment, typically by making measurements of prominent known landmarks, whether they be natural or artificial. The external sensors, however, do not tend to be as reliable as the dead reckoning sensors, so most navigation packages tend to use sensors of both types.

Figure 3 shows the area of the trial. The tunnel is approximate 250 meters long. The sensory data was collected while the LHD was running back and forward between the black circular marks.

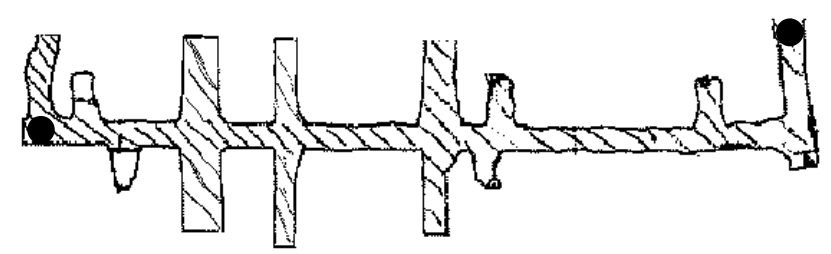

Figure 3. Map of the underground trial area.

\section{Dead Reckoning Sensors}

Two types of dead reckoning sensors were trialed; encoders and an Inertial Navigation System (INS). As dead reckoning sensors basically make measurements of the internal state of the vehicle, these sensors can typically be manufactured in a sealed unit, reducing the effect of environmental factors such as dust and water, both of which are highly prevalent in underground mining. For this reason, and the inherent robustness of such sensors, dead reckoning is normally used in any vehicle navigation system. This is particularly useful when data fusion algorithms such as the Kalman filter are employed as they tend to operate on a prediction-update cycle, where the prediction is generated via a vehicle model from dead reckoning sensors.

This section describes the data obtained from the INS and encoders systems during the field trials.

\subsection{Encoders}

Encoders are one of the most common sensors used to measure velocity or position of a rotating device.

Wheel rate encoders and steer angle encoders were passed through the particular vehicles forward kinematic equations to obtain an estimate of position and orientation. One problem with encoder based dead reckoning is that the vehicle model is usually derived in two dimensions, so the further the terrain is from a plane, the less accurate the solution. Another problem addressed in [3] is that encoder based dead reckoning cannot identify wheel slip. Encoders remain, though, as the cheapest way of obtaining dead reckoning information reliably. Figure 4 shows velocity and articulation angle during a period of 140 seconds of the run.
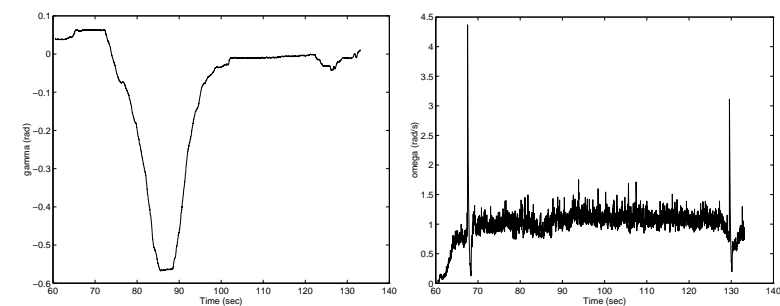

Figure 4. Steering angle and vehicle velocity

It should be noted that although encoders were used in this work, there are several other sensor types which may be used in an identical manner such as resolvers, potentiometers, and tachometers.

\subsection{Inertial Sensors}

The INS system used in the trials programme was a strapdown system consisting of a single triaxial accelerometer and four gyros. Two gyros are used for measuring roll and pitch rates. Two different gyros were used for measuring the rotation rate of the vehicle around vertical yaw. These two gyros are significantly more accurate and expensive than the roll and pitch gyros. The gyros were distributed in that form because in many applications yaw (heading) will be the only inertial sensor required. The Inertial sensors used were:

Accelerometer: An Entran triaxial acclaimer was used. This provides three analog outputs proportional to the acceleration of each of three orthogonal axes.

Roll (y) and Pitch (x) Gyros: Murata ENV05 solid state gyros were used. These sensors employ a vibrating piezoelectric prism which distorts and produces a voltage under the coriolis force generated by an angular velocity.

Andrews Fibre Optic Gyro: This is a single axis interferometric fibre optic gyroscope. This gyro is potentially very stable with drifts of less than $18 \mathrm{deg} / \mathrm{h}$ 
BAe Gyro. This is a vibrating structure type Gyro with very low drift characteristics.

These last two gyros were mounted along the vertical axis of the INS to provide measurements of orientation rate (yaw rate).

The complete INS system was mounted in a single sealed enclosure. A 12 bit AD converter and interface electronics were also mounted in this enclosure as shown in figure 5.

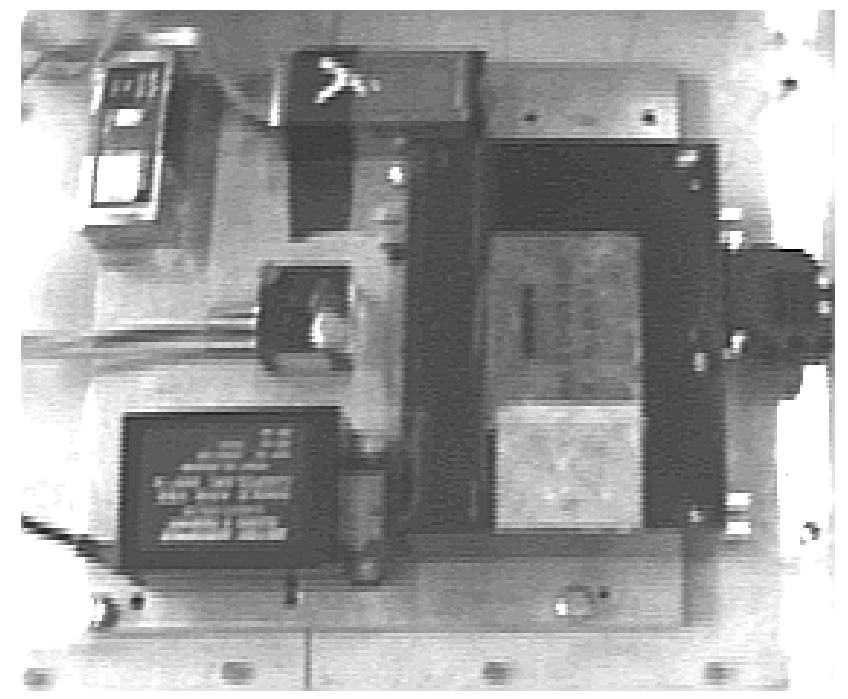

Figure 5. Strapdown INS platform.

A major advantage of inertial sensors is that they are non radiating and non jammable and may be packaged and sealed from the environment. This makes them potentially robust in the harsh environmental conditions common in underground mining. They have the potential of observing slip which is common in many under-ground mining machines.

The most common application of Inertial sensors is the use of a heading gyro. The integration of the gyro rate information provides the orientation of the vehicle.

Another application of Inertial sensors is the use of accelerometers to measure the attitude of the vehicle. The tilt of a platform can be evaluated with two orthogonal accelerometers and knowing the gravity value in the region of operation, assuming vehicle accelerations are negligible compared to gravity.

An INS system assembled from low cost solid-state components is almost always constructed in a "strapdown" configuration, as used in these trials. This means that all of the components, gyros and accelerometers are fixed to a common chassis and are not actively gimballed to align themselves in a pre-specified direction. This has the advantage of eliminating all moving parts. However the strap-down construction means that substantially more complex software is required to compute and distinguish true linear acceleration from angular acceleration and body roll or pitch with respect to gravity.

The first step in inertial navigation is aligning the platform to the navigation axes, that is initialising the axes in the strap-down platform. This can be done using the accelerometers installed in the platform [5]. If the platform is stationary we can assume that the accelerometers will measure the acceleration of gravity only. Then the attitude of the platform is determined with the accelerometers sensitive to $\mathrm{x}$ and $\mathrm{y}$ directions. The gyros and accelerometers usually drift with time and temperature. This bias must also be estimated by the alignment filter.

The Murata and BAE devices are known to be particularly sensitive to thermally induced drift. Built in thermal compensation is included in the Andrew device and later BAE devices. The standard deviation of the noise is also an important specification of the gyro. Due to the integration necessary to obtain heading a random walk angle will also be added. This angle is proportional to the standard deviation of the noise multiplied by the square root of time.

During operation, the Inertial Unit will accumulate errors with time. A calibration filter needs to be implemented to reduce the platform attitude errors. This calibration filter makes use of external information to reset the gyro information. The tilt information can also be be obtained directly from an accelerometer based tilt sensor. With an appropriate low pass filter these sensors will be sensitive mostly to gravity. This provides low frequency information of the platform attitude, while the high frequency component is provided by the gyros.

\subsection{Gyros}

The two best gyroscopes, the BAE gyro and the Andrews gyro, were used to record heading (yaw) data. Typical drift rates for the BAE and Andrew devices were 0.6 $\%$ min and $0.2 \%$ min respectively recorded over a 15 minute period. Non-linearities in the gyros result in errors in the measured rotations. This is especially prominent when the gyro is rotated and returned to the zero position. Typical accumulated errors for a $90^{\circ}$ excursion are $0.8^{\circ}$ for the BAE and $1.5^{\circ}$ for the Andrew.

Typical heading results for the BAE gyro and Andrews gyro are shown in Figures 6. The figure shows the vehicle starting out at zero degrees, turning a near ninety degree corner, stopping, reversing and turning (in reverse) back round the corner to the starting point. The gyros are mounted on the rear of the LHD articulation. As the LHD starts to turn, first corner in Figure 3, the rear articulation initially turns in the opposite direction before beginning the main turning operation (the front of the articulation will equally over-steer when coming out of the corner). This is clearly seen in the data at the start of the turn and at the end of the return corner.
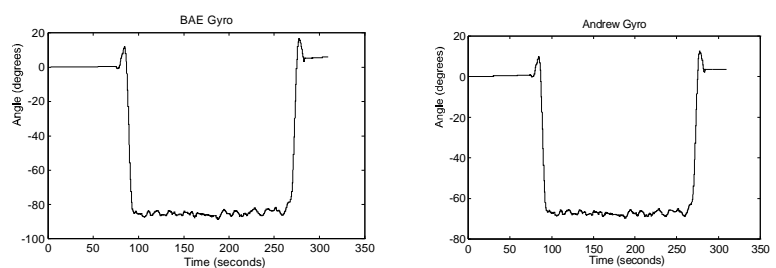

Figure 6: Heading (A) BAE gyro, (B) Andrews Gyro. 
As the LHD makes its way along the second straight, small variations in the heading direction can be seen. These variations are enlarged in Figure 12. From the plot we can see an oscillation of approximately 10 seconds that can be attributed to the driver correcting the heading of the vehicle. This heading change is about 2 degrees. There are also small amplitude variations in heading at higher frequencies. These variations can be attributed to the uneven terrain and wheel deformations.

Figure 7 shows typical roll and pitch angles recorded by integrating the output of the Murata gyros.
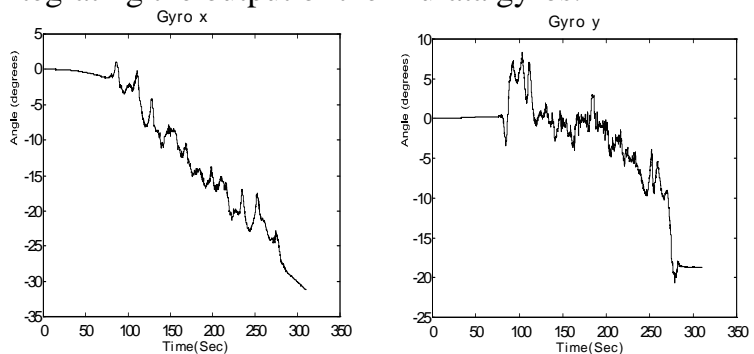

Figure 7: Murata Gyro x and y

These gyros are particularly sensitive to large zero offset errors. Static drift rates at a stable temperature are $0.5^{\circ} \% \mathrm{~min}$ and $1.3 \% \mathrm{~min}$ for the $\mathrm{X}$ and $\mathrm{Y}$ gyro's respectively. In addition these devices also suffer from some form of hysteresis with the zero offset varying to a new random value after motion. The offset varies by over $1 \% \mathrm{~min}$ from this source of error alone. The linearity of the Murata device also seems to be variable. Errors encountered calibrating the $\mathrm{Y}$ gyro varied between 0.5 and $3 \%$, the $\mathrm{X}$ gyro remaining below $1 \%$.

The attitude of the platform can be obtained integrating the measurements of roll, pitch and yaw angles from gyroscopes. The orientation angles must be estimated very accurately as even a small fraction of gravitational acceleration attributed to true linear acceleration results in huge errors

The gyros (BAe and Andrews) used for measuring angular rates around the vertical axis are sufficiently accurate to obtain yaw information. However, the roll and pitch gyros used in the INS package on the trials were not sufficiently accurate to provide sensible estimates of roll and pitch angles even over short periods of time.

\subsection{Accelerometers}

Measurements made by the accelerometers are in fact a sum of effects due to linear acceleration, orientation of the platform with respect to the gravity vector and angular acceleration of the platform as it moves round a curve. Figure 8 shows the signal output of the accelerometer with sensitive axis in the $\mathrm{x}$ direction. There are three distinct parts to the plot: The initial section 1 shows the acceleration measured while the LHD is stationary with the engine off. Almost no noise is present in this signal. Then the engine is started and the vibration is measured by the accelerometer 2 . The vehicle starts to move and the accelerometers measure vibration, acceleration due to tilt with respect to gravity, angular and linear acceleration 3 .

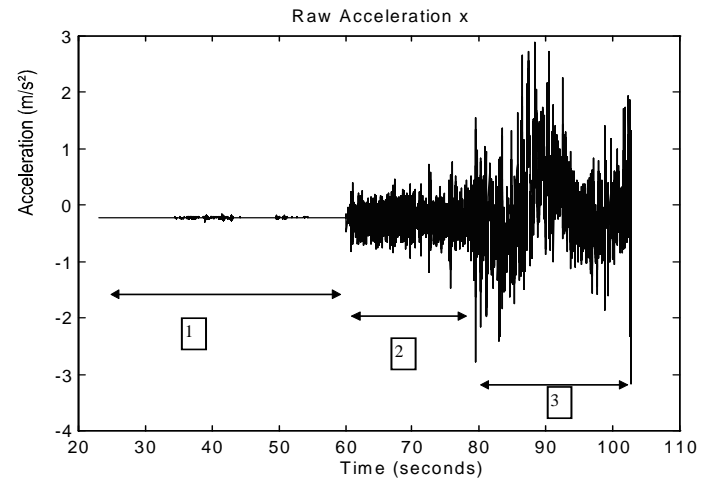

Figure 8: Acceleration measured along the y-axis

Two problems can be identified from these plots. First is that in order to use the accelerometer to predict position it is necessary to filter out acceleration due to the vibration of the vehicle. Second and most important of all, due to the small acceleration of the vehicle, the attitude of the platform must be obtained with high precision in order to compensate for the effect of gravity acceleration.

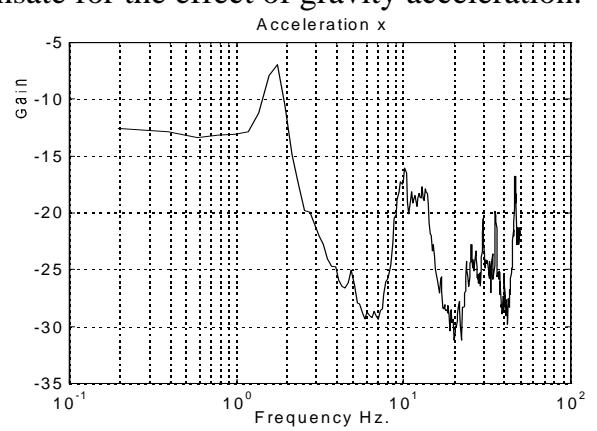

Figure 9: PSD of Acceleration information.

Figure 9 shows the Frequency components of the acceleration signal. It can be seen that engine vibration frequencies and harmonics are well identified over $7 \mathrm{~Hz}$. The main components are present below $2.5 \mathrm{hz}$ and can be attributed to acceleration due to the vehicle driver, uneven terrain and wheel deformation.

A third order low pass filter was designed and applied to the accelerometer data.
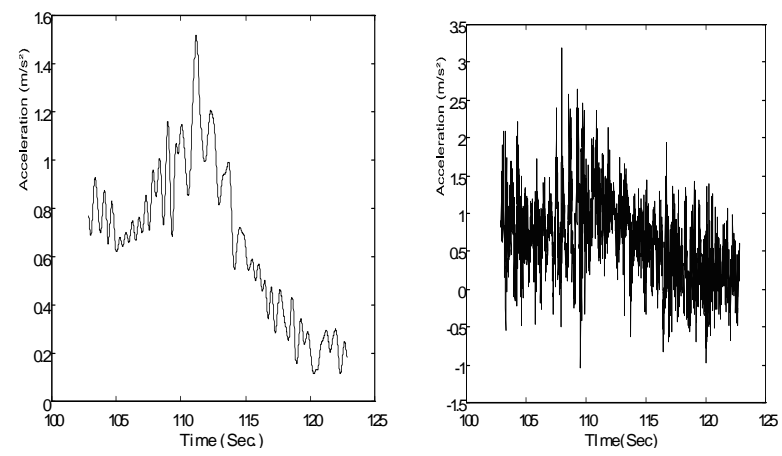

Figure 10: Filtered and Raw Acceleration

Figure 10 presents the filtered and raw data for acceleration $\mathrm{x}$, corresponding to 15 seconds of the run. It can be seen that the engine vibration has been eliminated. 
As expected vibration components closer to $1.5 \mathrm{~Hz}$. are still present. This is also detected by the gyros as can be seen in figures 11 and 12 .
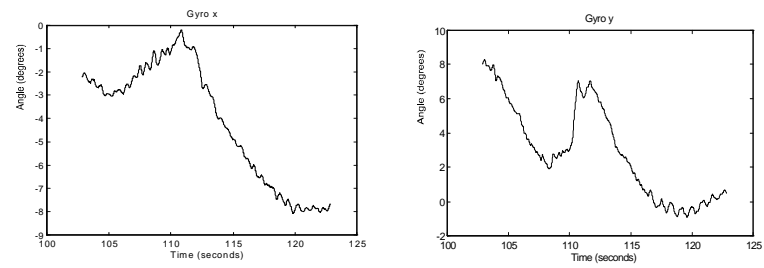

Figure 11: Murata Gyro x and y (15 Sec)

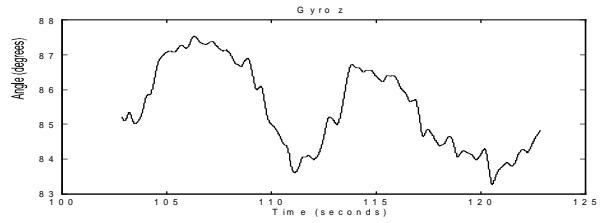

Figure 12: BAE z (15 Sec)

The accelerometer information can also be used to determine the attitude of the vehicle while moving. This will be useful to correct dead reckoning information while operating in 3-D environments.

\section{External Sensing}

\subsection{GCS Laser}

The GCS (Guidance Control Systems) laser is a commercially available sensor of a type widely used to solve to the positioning problem. The laser rotates horizontally at approximately $1 \mathrm{~Hz}$. scanning 360 degrees in azimuth. The rotating head has an encoder mounted, so the angle to a known retro-reflective target is the returned value. This laser is typically mounted on top of the vehicle, clear of obstructions. In indoor applications, where the speed of the vehicle is small compared to the target update rate, triangulation may be used without much loss in precision. This approach presents problems, however, when the vehicle has moved substantially between updates. In this case, a data fusion algorithm such as the Kalman filter must be employed.

From the trials, this sensor was found to be extremely reliable. Although the sensor was shock-mounted in order to reduce vibration due to vehicle motion, the only problem found was due to the vibration of the sensor. The problem, encountered only occasionally during high speed runs, is that the sensor appears to detect a target more than once, since the vibration of the vehicle induces a net (although very slight) backwards motion in the sensor. From evaluation using a Kalman filter explained in [3], the target match rate was approximately $90 \%$ which is good even for an indoor environment. Figure 13 shows the artificial landmark position and the path of the vehicle as estimated by the filter.

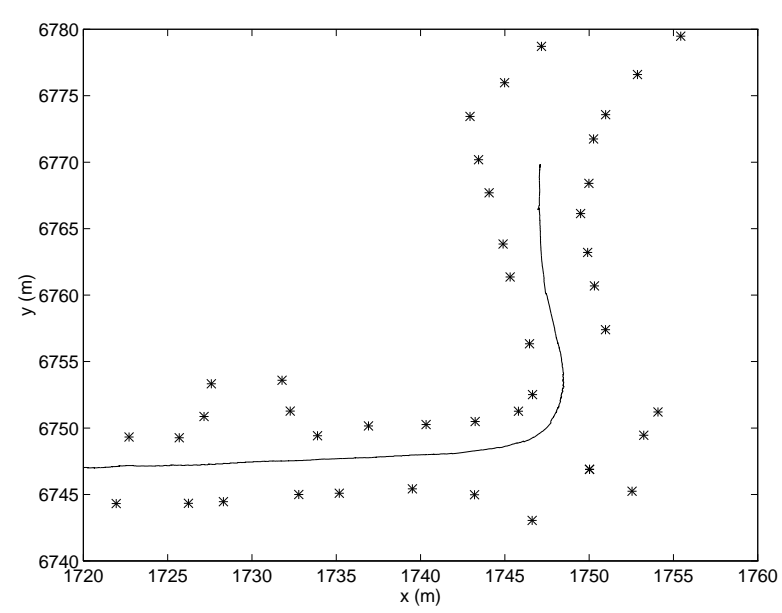

Figure 13. Beacon positions and filter path estimation.

\subsection{SICK Lasers}

These Lasers, produced by Erwin SICK optics, are timeof-flight devices, scanning 180 degrees in azimuth. They represent the first of a new wave of relatively cheap, but accurate profiling laser sensor. Two of these sensors were mounted on the LHD in an area clear of obstructions. The arrangement was such that an angle of about 270 degrees was covered by the sensors field of vision with a 90 degree overlap at the front of the vehicle.

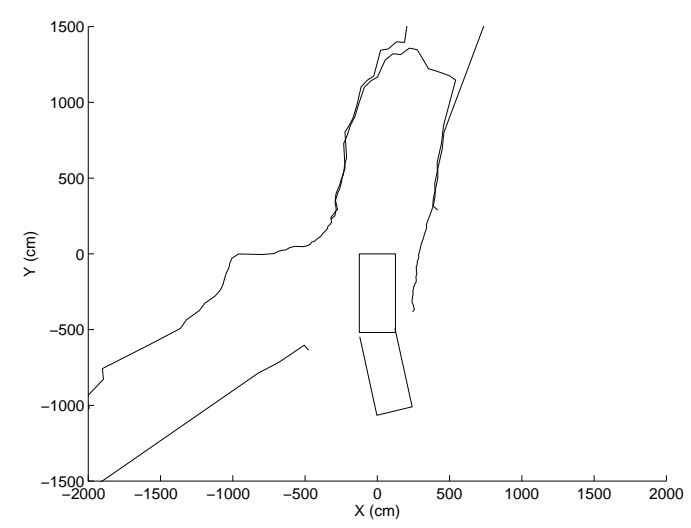

Figure 14: Laser profile of a mining tunnel

Figure 14 shows a typical profile given by the lasers. Even though the environment was extremely dusty, it can be seen that the profile of the tunnel walls is extremely good. Towards the end of the trials, a single SICK laser was pointed forwards and tilted down slightly (7.5 degrees). This small amount of tilt, combined with the motion of the vehicle, allows a three-dimensional model of the tunnel to be created. A 3-D model is potentially useful when considering the collision avoidance problem.

Figure 15 shows two 3-D representations of the tunnel from two separate runs. The top plot shows a typical section of the test tunnel. It is clear that the tunnel floor is relatively smooth and that the walls are fairly flat. A side tunnel can be seen to the left. The bottom plot shows the 
tunnel profile immediately in front of a muck-pile. It is easy to see that there are small boulders/rocks scattered about the tunnel floor, and that the tunnel walls are much rougher. Muck pile shape characterisation would be useful for planning automated digging and for measuring rock fragmentation.

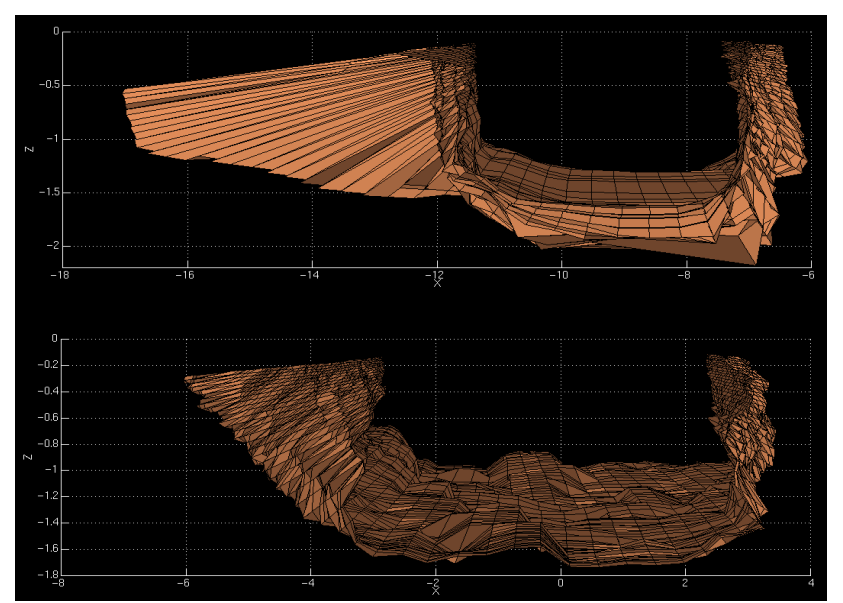

Figure 15: 3-D tunnel maps. Top: typical tunnel. Bottom: section

As can be seen from the data, the SICK laser sensor promises to provide an extremely good way of identifying tunnel features. Whether this information would be used to build maps, or match the observed data to a stored map is application dependant, though having a stored map will reduce the risk of spurious readings causing the navigation system to fail. It should be noted that since the SICK laser operates using a rotating mirror, long term exposure to vibration may cause untimely degradation in performance. Also, the life of laser diodes such as the one used are known to reduce with exposure to elevated temperatures such as those found in underground mines.

\subsection{Ultrasonics}

Ultrasonic sensors are also time-of-flight devices used widely for many applications. These sensors come in many shapes and forms, only some of which are suitable for use in an underground environment.

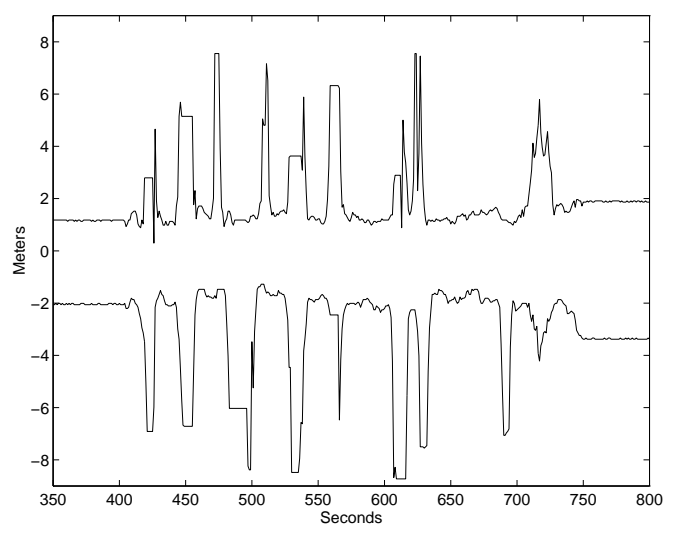

Figure 16: Ultrasonic tunnel profile
Sensors such as the Polaroid family of ultrasonic transducer are typically very reliable, but suffer in a dusty environment as the vibrating element is open to the environment It is therefore recommended to use a sealed unit, easily cleanable, such as MASA's family of ceramic ultrasonic transducers. The value of the ultrasonic sensors as a navigation aid is in their ability to detect gross changes in the features of the tunnel as shown in figure 16. This data may be used to detect side tunnels and similar features, using map matching techniques.

\section{Conclusions}

This paper presented the results from an extensive trial of commercially available sensors in a harsh underground mining environment. On the whole, all of the sensors worked better and more reliably than was initially expected. A further long-term trial is needed to determine sensor degradation patterns and failure modes.

From the results, two main navigation solutions have presented themselves, which are constrained by the wish for low infrastructure. The first is a geometric solution in which encoder based dead reckoning is fused with periodic updates to artificial landmarks using a sensor such as the GCS laser. This solution requires infrastructure, though far less than existing systems. The second solution is one that is feature based. INS based dead reckoning is fused with sensor information of natural landmarks, such as the SICK laser or ultrasonics.

Ideally, the two navigation systems will operate in parallel, providing robustness through redundancy. The inherent robustness is further enhanced through the use of different sensing technologies for each navigation system, as each separate sensor will have unique failure modes. In systems which use double or triple redundancy of a single sensor, the failure modes of the sensors may be identical and therefore fail simultaneously.

\section{References}

[1] F. Labonte, J., Giraud L., and Polotski V., "Telerobotics issues in the operation of a LHD vehicle", Proceedings of the Third Canadian Conference on Computer Applications in the Mineral Industry, 1995, pp 672-681

[2] Makela H., Lehtinen H, Rintanen K., Koskinen K., Navigation System for LHD machines", Intelligent Autonomous Vehicles 95. Finland, pp. 314-319, 1995.

[3] S. Scheding, G. Dissanayake, E. Nebot and H. Durrant-Whyte, "Slip Modelling and Aided Inertial Navigation of an LHD", submitted to IEEE Conference on Robotics and Automation, 1997

[4] J. Steele, C. Ganesh, and A. Kleve, "Control and Scale Model Simulation of Sensor-Guided LHD Mining Machines", IEEE Transactions on Industry Applications, 1993, pp 1232-1238

[5] Fuke Y., Krotkov E., "Dead reckoning for a lunar rover on uneven terrain", IEEE Int. Conf. Robotics and Automation, Minneapolis, Minessota, 1996., pp. 411-416. 